Equilibrated Patch Recovery error estimates: simple and accurate upper bounds of the error, Diez, P., Rodenas, J.J. and Zienkiewicz, O.C., International Journal for Numerical Methods in Engineering, Vol. 69, Issue 10, pp. 2075-2098, 2007

\title{
Equilibrated Patch Recovery error estimates: simple and accurate upper bounds of the error
}

\author{
Pedro Díez*, Juan José Ródenas ${ }^{\dagger}$ and Olgierd C. Zienkiewicz ${ }^{\S}$ \\ * LaCaN, Universitat Politècnica de Catalunya, Barcelona, Spain \\ $\dagger$ Departamento de Inteniería Mecánica y de Materiales \\ Universidad Politécnica de Valencia, Spain \\ $\S$ School of Engineering, University of Wales, Swansea, UK
}

\begin{abstract}
SUMMARY
This paper introduces a new recovery-type error estimator ensuring local equilibrium and yielding a guaranteed upper bound of the error. The upper bound property requires the recovered solution to be both statically equilibrated and continuous. The equilibrium is obtained locally (patch by patch) and the continuity is enforced by a postprocessing based on the partition of the unity concept. This postprocess is expected to preserve the features of the locally equilibrated stress field. Nevertheless, the postprocess phase modifies the equilibrium, which is no longer exactly fulfilled. A new methodology is introduced that yields upper bound estimates by taking into account this lack of equilibrium. This requires computing the $\mathcal{L}_{2}$ norm of the error or relating it with the energy norm.

The guaranteed upper bounds are obtained by using a pessimistic bound of the error $\mathcal{L}_{2}$ norm, derived from an eigenvalue problem. Nevertheless, these bounds are not sharp. An additional strategy based on a more accurate assessment of the error $\mathcal{L}_{2}$ norm is introduced, providing sharp estimates which are practical upper bounds as it is demonstrated in the numerical tests.
\end{abstract}

KEY WORDS: Error estimates, Stress recovery, Equilibrated stresses, Upper bounds

\section{Introduction}

The error assessment tools for Finite Element analysis are classified into two families: residualtype error estimators and recovery-based error estimators. This classification is widely accepted and already used in textbooks on error estimation $[1,2]$.

The recovery-based estimates, based on the ideas of Zienkiewicz and Zhu [3, 4, 5, 6], are often preferred by practitioners, due to their simple implementation and robustness $[7,8,9]$.

On the other hand, the a posteriori implicit residual-type estimators have a sounder mathematical basis and produce estimates that are guaranteed upper or lower bounds of the error $[1,10,11]$. In particular, the upper bound property is a consequence of the equilibrated stress field resulting from the implicit residual approach. The ability to produce guaranteed

* Correspondence to: Campus Nord UPC, Módulo C-2, Jordi Girona 1, E-08034 Barcelona, Spain 
upper and lower bounds of the energy norm of the error is especially desirable in the context of goal-oriented adaptivity.

The idea of enforcing equilibrium in the recovery procedure has been used by several authors $[12,13,14,15,16,17,18,19,20,21,22,23,24,25]$. Originally, the equilibrium was imposed locally to enhance the least-squares fitting and improve the nodal values of the recovered stresses. The resulting smoothed stresses are however not equilibrated and therefore the upper bound property is not ensured. The idea of producing a statically admissible (equilibrated and continuous) stress field using a recovery technique was used also by Kvamsdal and Oksad in [22]. However, the error estimate presented there is not a rigorous upper bound. On the one hand, due to the process used to ensure continuity, the resulting recovered stresses no longer satisfy the internal equilibrium equation (Kvamsdal and Oksad assume in [22] that the effects on the estimate of the lack of equilibrium introduced when enforcing continuity are negligible). On the other hand, these authors only obtained a low quality approximate satisfaction of the natural boundary conditions as they simply considered the minimization of the residual of the boundary equilibrium equation. In the present work the equilibrium is enforced following a different approach. The local recovery introduces the exact satisfaction of the imposed tractions along the Neumann boundary and the interior pointwise equilibrium via the Lagrange multipliers technique, see [24, 25]. In order to improve the quality of the recovered stress field, the compatibility conditions are also enforced in the reast-squares projections as

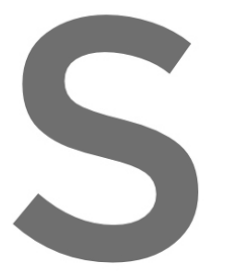
an additional linea true upper bounds stress continuity is the $\mathcal{L}_{2}$ norm of the norm using the solv and, consequently,


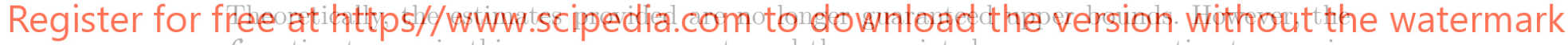
$\mathcal{L}_{2}$ estimates are in this case very accurate and the associated energy norm estimates are, in practice, upper bounds. The numerical tests corroborate this claim.

The remainder of the paper is organized as follows. Section 2 briefly introduces the model problem. The error equations and bounds are stated in section 3 . Then, section 4 is devoted to describe a recovery technique enforcing equilibrium and a postprocessing enforcing continuity. This generates nearly Statically Admissible recovered stresses. The strict upper bounds are then computed accounting for the lack of equilibrium introduced when enforcing continuity. The numerical examples included in section 5 demonstrate that the proposed strategy furnishes sharp and reliable practical upper bound error estimates. 


\section{Problem statement}

Let us consider the linear elasticity problem. The unknown displacement field $\boldsymbol{u}$, taking values in $\Omega \subset \mathbb{R}^{d}$ (with $d$ the number of spatial dimensions, $d=1,2$ or 3 ), is the solution of the boundary value problem

$$
\begin{aligned}
-\nabla \cdot \boldsymbol{\sigma}(\boldsymbol{u}) & =\boldsymbol{b} & & \text { in } \Omega \\
\boldsymbol{\sigma}(\boldsymbol{u}) \cdot \boldsymbol{n} & =\boldsymbol{t} & & \text { on } \Gamma_{N} \\
\boldsymbol{u} & =\mathbf{0} & & \text { on } \Gamma_{D}
\end{aligned}
$$

where $\Gamma_{N}$ and $\Gamma_{D}$, with $\partial \Omega=\overline{\Gamma_{N}} \cup \overline{\Gamma_{D}}$ and $\Gamma_{N} \cap \Gamma_{D}=\emptyset$, are the Neumann and Dirichlet parts of the boundary. The Dirichlet boundary condition (1c) is taken homogeneous in the developments for the sake of simplicity.

The weak form of the problem reads: find $u \in \mathcal{V}$ such that

$$
a(\boldsymbol{u}, \boldsymbol{v})=l(v), \text { for all } \boldsymbol{v} \in \mathcal{V},
$$

where $\mathcal{V}$ is the standard test space for the elasticity problem, $\mathcal{V}=\left[\mathcal{H}_{\Gamma_{0}}^{1}(\Omega)\right]^{d}$
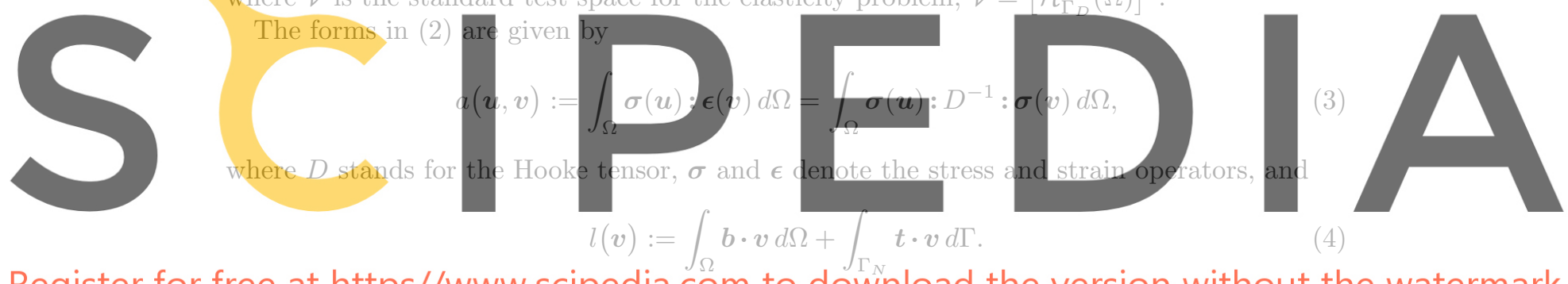

Register for free at https//www.scipedia.com to download the version without the watermark

The energy bilinear form $a(\cdot, \cdot)$ is expressed also in terms of stresses instead of displacements

by formally introducing $\bar{a}(\cdot, \cdot)$ such that

$$
\bar{a}(\boldsymbol{\sigma}, \boldsymbol{\tau}):=\int_{\Omega} \boldsymbol{\sigma}: D^{-1}: \tau d \Omega
$$

Note that, with this definition, $a(\boldsymbol{u}, \boldsymbol{v})=\bar{a}(\boldsymbol{\sigma}(\boldsymbol{u}), \boldsymbol{\sigma}(\boldsymbol{v}))$.

3. Discrete approximation and error bounding

Let $\boldsymbol{u}^{H}$ be a finite element approximation to $\boldsymbol{u}$. The solution $\boldsymbol{u}^{H}$ lies in a functional space $\mathcal{V}^{H} \subset \mathcal{V}$, associated with a mesh of isoparametric finite elements of characteristic size $H$, and it is such that

$$
a\left(\boldsymbol{u}^{H}, \boldsymbol{v}\right)=l(\boldsymbol{v}), \text { for all } \boldsymbol{v} \in \mathcal{V}^{H} .
$$

Note that the mesh is not necessarily conforming. Non-conforming meshes are used for the examples in section 5 and the continuity required by the condition $\mathcal{V}^{H} \subset \mathcal{V}$ is enforced using local constraints, often called multi-point constraints(MPC's) [26, 27], at the non-conforming (hanging) nodes. 


\subsection{Error assessment}

We focus on assessing the error $\boldsymbol{e}:=\boldsymbol{u}-\boldsymbol{u}^{H}$ measured in the energy norm, induced by $a(\cdot, \cdot)$ and denoted by $\|\cdot\|$. That is, the quantity to be assessed is $\|\boldsymbol{e}\|^{2}=a(\boldsymbol{e}, \boldsymbol{e})=\bar{a}\left(\boldsymbol{\sigma}_{e}, \boldsymbol{\sigma}_{e}\right)$, where $\boldsymbol{\sigma}_{e}:=\boldsymbol{\sigma}(\boldsymbol{u})-\boldsymbol{\sigma}\left(\boldsymbol{u}^{H}\right)$.

Each component of the approximate stress field $\boldsymbol{\sigma}\left(\boldsymbol{u}^{H}\right)$ lies in the functional space of the derivatives of the displacements in $\mathcal{V}^{H}$. Consequently, $\boldsymbol{\sigma}\left(\boldsymbol{u}^{H}\right)$ is generally discontinuous across element edges but smooth inside the elements. A recovered stress field $\boldsymbol{\sigma}^{\star}$ is sought in a richer functional space $\mathcal{S}$. The original approaches to recovery-type error estimates select $\mathcal{S}$ equal to $\mathcal{V}^{H}$, in the sense that each component of the stresses in $\mathcal{S}$ lies in the same space of each component of the displacements in $\nu^{H}$. Then $\sigma^{*}$ is determined as a least-squares fitting of $\sigma\left(u^{H}\right)$ in $\mathcal{S}$.

Once $\sigma^{\star}$ is computed, the error estimate is readily obtained by assuming that $\sigma^{\star}$ is a good approximation to $\sigma(\boldsymbol{u})$ :

$$
\sigma_{e}^{\star}=\sigma^{\star}-\sigma\left(u^{H}\right) .
$$

Then $\sigma_{e}^{\star}$ is a good approximation of $\sigma_{e}$ and hence the corresponding energy norm estimate is $\bar{a}\left(\sigma_{e}^{\star}, \sigma_{e}^{\star}\right) \approx\|e\|^{2}$. This is the main rationale for the recovery-type error estimates introduced by Zienkiewicz and Zhu $[3,4,5,6]$.


In order to obtain a computable assessment of the error, this equation has to be stated loc:

Register for free at https//www.scipedia.com to download the version without the watermark 3.2. Statically admissible stresses and error upper bound

The residual-type error estimators build up an statically admissible (locally equilibrated and continuous) stress field and furnish upper bounds of the energy norm of the error [1, 22]. This property is also obtained if the statically admissible stresses are obtained after a recovery procedure by properly imposing the equilibrium restrictions.

Proposition 1. Let $\Omega$ be an open bounded domain and let $\Omega_{k}, k=1, \ldots, \mathrm{n}_{\mathrm{elem}}$, be the elements of a finite element mesh of characteristic size $H$ discretizing $\Omega$. Let $\boldsymbol{\sigma}^{\star}$ be a statically admissible stress field, that is, continuous and locally equilibrated:

$$
\begin{array}{rr}
-\nabla \cdot \boldsymbol{\sigma}^{\star}=\boldsymbol{b} & \text { in } \Omega_{k} \text { for } k=1, \ldots, \mathrm{n}_{\mathrm{elem}} \\
\boldsymbol{\sigma}^{\star} \cdot \boldsymbol{n}=\boldsymbol{t} & \text { on } \Gamma_{N} \cap \partial \Omega_{k} \text { if non-empty }
\end{array}
$$

Then $\boldsymbol{\sigma}^{\star}$ is such that

$$
\bar{a}\left(\boldsymbol{\sigma}^{\star}, \boldsymbol{\sigma}(\boldsymbol{v})\right)=l(\boldsymbol{v}), \text { for all } \boldsymbol{v} \in \mathcal{V} .
$$

Proof. The proof uses the classical weighted residuals technique locally in each $\Omega_{k}$ and then continuity of $\boldsymbol{\sigma}^{\star}$ in order to cancel the stress jumps resulting in assembling all elementary contributions. 
Proposition 2. Let $\boldsymbol{\sigma}^{\star}$ be a statically admissible stress field, then the associated energy measure is larger than the energy norm of the exact solution of the elasticity problem, $\boldsymbol{u}$. That is

$$
\|\boldsymbol{u}\|^{2}=\bar{a}(\boldsymbol{\sigma}(\boldsymbol{u}), \boldsymbol{\sigma}(\boldsymbol{u})) \leq \bar{a}\left(\boldsymbol{\sigma}^{\star}, \boldsymbol{\sigma}^{\star}\right) .
$$

Proof. Taking $\boldsymbol{v}=\boldsymbol{u}$ in (10) and using (2),

$$
\bar{a}\left(\boldsymbol{\sigma}^{\star}, \boldsymbol{\sigma}(\boldsymbol{u})\right)=l(\boldsymbol{u})=\bar{a}(\boldsymbol{\sigma}(\boldsymbol{u}), \boldsymbol{\sigma}(\boldsymbol{u}))=\|u\|^{2} .
$$

Then, the proof is completed using the Cauchy-Schwartz inequality

$$
\sqrt{\bar{a}\left(\sigma^{\star}, \sigma^{\star}\right)}\|u\| \geq\|u\|^{2} \text {. }
$$

The next proposition states that the upper bound property holds also for the error estimate when the recovered stress field $\sigma^{\star}$ is statically admissible.

Proposition 3. Let $\sigma_{e}^{\star}:=\sigma^{\star}-\sigma\left(u^{H}\right)$ be a stress error estimate such that the recovery stresses $\sigma^{\star}$ are statically admissible. Then, the associated energy error estimate is an upper bound of the actual energy error. That is
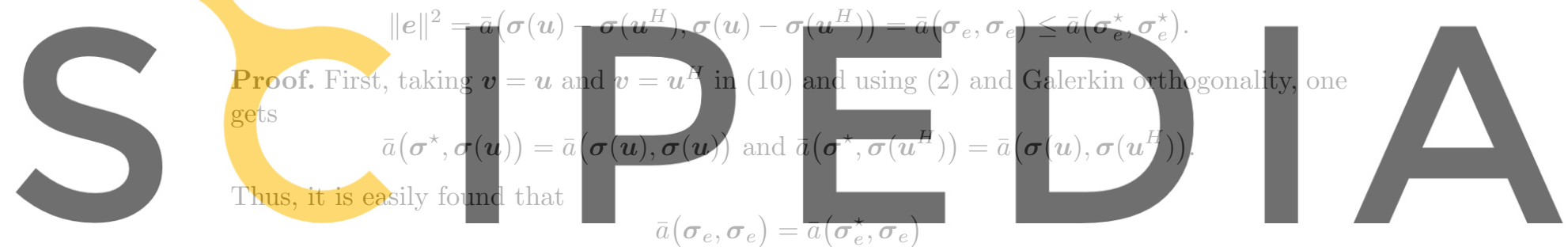

Register for free at hetest of $/$ he proof is analpgous to the prgvious pne. the version without the watermark

\subsection{Nearly statically admissible stresses and related upper bounds}

Assume that $\boldsymbol{\sigma}^{\star}$ is a nearly statically admissible stress field. This means that either the continuity or the equilibrium are not strictly fulfilled.

In the first case, $\boldsymbol{\sigma}^{\star}$ verifies (9) but the traction jump across the set of all internal edges $\Gamma_{\text {int }}, \llbracket \boldsymbol{\sigma}^{\star} \cdot \boldsymbol{n} \rrbracket_{\Gamma_{\text {int }}}$ does not vanish. The jump $\llbracket \boldsymbol{\sigma}^{\star} \cdot \boldsymbol{n} \rrbracket_{\Gamma_{\text {int }}}$ is formally defined as

$$
\llbracket \boldsymbol{\sigma}^{\star} \cdot \boldsymbol{n} \rrbracket_{\Gamma_{\mathrm{int}}}=\left(\left.\boldsymbol{\sigma}^{\star}\right|_{\Omega_{1}}-\left.\boldsymbol{\sigma}^{\star}\right|_{\Omega_{2}}\right) \cdot \boldsymbol{n}
$$

where $\Omega_{1}$ and $\Omega_{2}$ are the two elements separated by $\Gamma_{\text {int }}$ (that is $\Gamma_{\text {int }}=\overline{\Omega_{1}} \cap \overline{\Omega_{2}}$ ) and such that $\boldsymbol{n}$ is pointing from $\Omega_{1}$ to $\Omega_{2}$ (outward normal to $\Omega_{1}$ ). Note that this definition is independent of the choice of $\Omega_{1}$ and $\Omega_{2}$, that is, of the sign of $\boldsymbol{n}$.

The second case refers to a continuous stress field $\boldsymbol{\sigma}^{\star}$ verifying (9b) and a (slightly) modified version of $(9 \mathrm{a})$, that is

$$
-\nabla \cdot \sigma^{\star}=b+s .
$$

In both cases the equilibrium defects are assumed to be small compared with the corresponding quantities, that is $\|\boldsymbol{s}\| \lll\|\boldsymbol{b}\|$ and $\llbracket \boldsymbol{\sigma}^{\star} \cdot \boldsymbol{n} \rrbracket_{\Gamma_{\text {int }}}$ small compared with the average values of $\boldsymbol{\sigma}^{\star}$.

The upper bound properties have to be modified to include these equilibrium defects. 
Proposition 4. Let $\boldsymbol{\sigma}^{\star}$ be a locally equilibrated stress field, that is verifying (9), not continuous across the interelement edges $\Gamma_{\text {int }}$. The jump of the traction across $\Gamma_{\text {int }}$ is denoted by $\llbracket \boldsymbol{\sigma}^{\star} \cdot \boldsymbol{n} \rrbracket_{\Gamma_{\text {int }}}$.

Then $\boldsymbol{\sigma}^{\star}$ is such that

$$
\bar{a}\left(\boldsymbol{\sigma}^{\star}, \boldsymbol{\sigma}(\boldsymbol{v})\right)=l(\boldsymbol{v})+\int_{\Gamma_{\text {int }}} \boldsymbol{v} \cdot \llbracket \boldsymbol{\sigma}^{\star} \cdot \boldsymbol{n} \rrbracket_{\Gamma_{\text {int }}} d \Gamma, \text { for all } \boldsymbol{v} \in \mathcal{V} .
$$

Moreover, the energy norm of $\boldsymbol{\sigma}^{\star}$ is such that

$$
\|u\|^{2} \leq \bar{a}\left(\sigma^{*}, \sigma^{\star}\right)-2 \int_{\Gamma_{\text {int }}} u \cdot \llbracket \sigma^{\star} \cdot n \rrbracket_{\Gamma_{\text {int }}} d \Gamma
$$

and the corresponding error estimate, computed from $\sigma_{e}^{\star}:=\sigma^{\star}-\sigma\left(\boldsymbol{u}^{H}\right)$, is such that

$$
\|e\|^{2} \leq \bar{a}\left(\sigma_{e}^{\star}, \sigma_{e}^{\star}\right)-2 \int_{\Gamma_{\text {int }}} e \cdot \llbracket \sigma^{\star} \cdot n \rrbracket_{\Gamma_{\text {int }}} d \Gamma .
$$

Proof. (12) is the standard weak form for discontinuous stresses. The expressions for the



upper bounds (13)

The following resul equilibrated.

Proposition 5. L

(9a), that is, such that


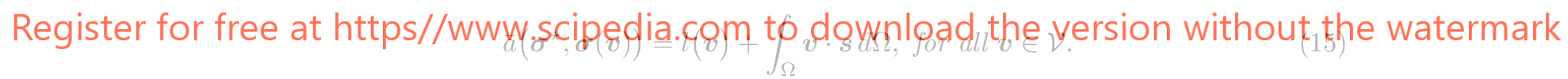

Moreover, the energy norm of $\boldsymbol{\sigma}^{\star}$ is such that

$$
\|\boldsymbol{u}\|^{2} \leq \bar{a}\left(\boldsymbol{\sigma}^{\star}, \boldsymbol{\sigma}^{\star}\right)-2 \int_{\Omega} \boldsymbol{u} \cdot \boldsymbol{s} d \Omega
$$

and the corresponding error estimate, computed from $\boldsymbol{\sigma}_{e}^{\star}:=\boldsymbol{\sigma}^{\star}-\boldsymbol{\sigma}\left(\boldsymbol{u}^{H}\right)$ is such that

$$
\|\boldsymbol{e}\|^{2} \leq \bar{a}\left(\boldsymbol{\sigma}_{e}^{\star}, \boldsymbol{\sigma}_{e}^{\star}\right)-2 \int_{\Omega} \boldsymbol{e} \cdot \boldsymbol{s} d \Omega .
$$

Proof. (15) is standard, then the proof is practically identical to proposition 4

Remark 1. Note that the upper bounds given by (13), (14), (16) and (17) are not computable because the integral terms in the r.h.s. involve the unknowns $\boldsymbol{u}$ and $\boldsymbol{e}$.

In the following, the result given in proposition 5 is exploited to obtain a strict upper bound of the energy error from recovered stresses that are not exactly statically admissible. An analogous procedure using proposition 4 is not included in this paper. 
4. A nearly statically admissible stress recovery technique

In order to obtain an upper bound of the error, it suffices that the recovery technique yields a statically admissible recovered stress field $\boldsymbol{\sigma}^{\star}$. As stated above, this requires the stress field to be locally equilibrated and continuous. It has been also noted in the previous section that if these conditions are not strictly fulfilled the resulting expressions must account for either the continuity or the equilibrium defects, see (14) and (17).

This section introduces practical recovery techniques that meet these conditions and therefore are used to produce upper bound error estimates.

\subsection{Locally equilibrated stress recovery}

The nodal points of the mesh are denoted by $P_{i}\left(i=1, \ldots, \mathrm{n}_{\text {poin }}\right.$, with $\mathrm{n}_{\text {poin }}$ the number of nodal vertices in the mesh). The patch of elements surrounding $P_{i}$ is denoted by $\omega_{i}$ and $N_{i}$ stands for the lineal shape function associated with the vertex node. Note that $\omega_{i}$ is precisely the support of $N_{i}$.

Remark 2. If the elements are of order larger than one, the linear shape functions $N_{i}$ and the actual shape functions used in the functional approximation do not coincide.


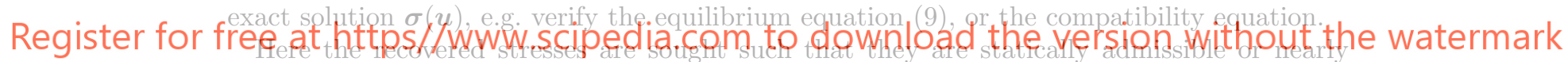

statically admissible in order to derive upper bound error estimates.

Imposing the equilibrium condition (9) to the polynomial stress field $\sigma_{i}^{*}$ results on adding linear constraints to the local least-squares fitting. This is easily done by using the SPR-C technique [25], which is a modification of the standard SPR technique. In the SPR-C technique, the equilibrium condition (9) is imposed to the polynomial stress field $\boldsymbol{\sigma}_{i}^{\star}$ by applying a set of linear constraints via the Lagrange multiplier technique.

Thus, the local recovered stresses $\boldsymbol{\sigma}_{i}^{\star}$ are computed imposing the additional condition (9), that is

$$
-\nabla \cdot \boldsymbol{\sigma}_{i}^{\star}=\boldsymbol{b} \text { in } \omega_{i} \quad \text { and } \quad \boldsymbol{\sigma}_{i}^{\star} \cdot \boldsymbol{n}=\boldsymbol{t} \text { on } \Gamma_{N} \cap \partial \omega_{i} \text { if non-empty. }
$$

Moreover, in order to further improve the quality of the recovered stress filed, the compatibility condition may also be imposed. The compatibility condition relates the derivatives of the stress components and ensures that the stress tensor corresponds to a kinematically admissible displacement field via the linear elastic constitutive relation. For instance, in the case of the plane stress state it reads (see [29])

$$
\frac{\partial^{2}}{\partial y^{2}}\left(\sigma_{x}-\nu \sigma_{y}\right)+\frac{\partial^{2}}{\partial x^{2}}\left(\sigma_{y}-\nu \sigma_{x}\right)=2(1+\nu) \frac{\partial^{2} \tau_{x y}}{\partial x \partial y},
$$

which is an additional linear constraint to the least-squares projection. 


\subsection{Continuous recovered stresses}

In order to impose continuity in the representation of the recovered stresses, the following expression is used:

$$
\boldsymbol{\sigma}^{\star}=\sum_{i=1}^{\mathrm{n}_{\mathrm{poin}}} N_{i} \boldsymbol{\sigma}_{i}^{\star}
$$

Consequently, the proposed representation for $\sigma^{\star}$ is automatically continuous and due to the $\delta$ property of the shape functions $\left(N_{i}\left(P_{j}\right)=\delta_{i j}\right)$ it is found that $\boldsymbol{\sigma}^{\star}\left(P_{i}\right)=\boldsymbol{\sigma}_{i}^{\star}\left(P_{i}\right)$ for every $i=1, \ldots, \mathrm{n}_{\text {poin }}$.

Remark 3. Note that the fitted polynomial stresses $\sigma^{\star}$ are used in all the points of the patch $\omega_{i}$. This contrasts with the original SPR techniques (equilibrated or not) where only the value of $\sigma_{i}^{\star}$ in $P_{i}$ is used. In fact, equation (19) must be read as

$$
\sigma^{\star}(x)=\sum_{i=1}^{\mathrm{n}_{\text {poin }}} N_{i}(\boldsymbol{x}) \sigma_{i}^{\star}(\boldsymbol{x})
$$


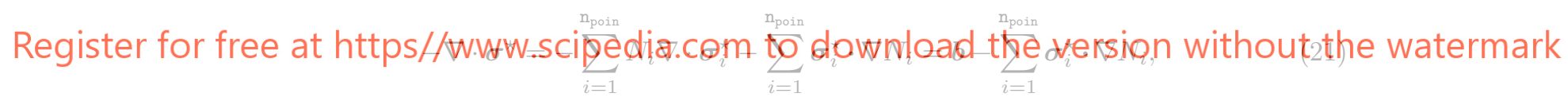

using (18) and the fact that $\sum_{i=1}^{\mathrm{n}_{\mathrm{poin}}} N_{i}=1$. Note that the recovered stress $\boldsymbol{\sigma}^{\star}$ is not equilibrated because of the term $\sum_{i=1}^{\mathrm{n}_{\text {poin }}} \boldsymbol{\sigma}_{i}^{\star} \cdot \nabla N_{i}$.

As already mentioned, the hypothesis behind the SPR philosophy is that $\left.\boldsymbol{\sigma}_{i}^{\star} \approx \boldsymbol{\sigma}(\boldsymbol{u})\right|_{\omega_{i}}$. In every element $\Omega_{k}, k=1, \ldots, \mathrm{n}_{\text {elem }}$, there are $\mathrm{n}_{\text {node }}$ (with $\mathrm{n}_{\text {node }}$ the number of element vertex nodes, 3 for triangles, 4 for quadrilaterals...) different stress fields that are supposed to be good approximations of $\left.\boldsymbol{\sigma}(\boldsymbol{u})\right|_{\Omega_{k}}$. Thus, the average recovered stress $\boldsymbol{\sigma}_{A}^{\star}$ is introduced by defining its restriction to $\Omega_{k}$

$$
\left.\boldsymbol{\sigma}_{A}^{\star}\right|_{\Omega_{k}}=\left.\frac{1}{\mathrm{n}_{\text {node }}} \sum_{P_{i} \in \Omega_{k}} \boldsymbol{\sigma}_{i}^{\star}\right|_{\Omega_{k}}, \text { for } k=1,2, \ldots, \mathrm{n}_{\mathrm{elem}} .
$$

Note that $\boldsymbol{\sigma}_{A}^{\star}$ is defined almost everywhere (except on the interelement edges) in $\Omega$, it is continuous inside the elements and globally discontinuous. Thus, equation (21) results on

$$
-\nabla \cdot \boldsymbol{\sigma}^{\star}=\boldsymbol{b}+\sum_{i=1}^{\mathrm{n}_{\text {poin }}}\left(\boldsymbol{\sigma}_{A}^{\star}-\boldsymbol{\sigma}_{i}^{\star}\right) \cdot \nabla N_{i}-\underbrace{\sum_{i=1}^{\mathrm{n}_{\mathrm{poin}}} \boldsymbol{\sigma}_{A}^{\star} \cdot \nabla N_{i}}_{=0}
$$


where the last term of the r.h.s. vanishes because $\boldsymbol{\sigma}_{A}^{\star}$ does not depend on $i$ and $\sum_{i=1}^{\mathrm{n}_{\text {poin }}} \nabla N_{i}=\mathbf{0}$. The term

$$
\boldsymbol{s}=\sum_{i=1}^{\mathrm{n}_{\text {poin }}}\left(\boldsymbol{\sigma}_{A}^{\star}-\boldsymbol{\sigma}_{i}^{\star}\right) \cdot \nabla N_{i}
$$

is the equilibrium defect introduced by the continuity requirement. If the recovered stresses are indeed very similar in the overlapping patches, $s$ must be small. In fact, Kvamsdal and Okstad [22] claim that this term is negligible and they consider $\boldsymbol{\sigma}^{\star}$ to be statically admissible.

In the following, the equilibrium defect $s$ is accounted for in a strict upper bound estimate.

\subsection{Upper bound estimate from the continuous recovered stresses}

The global continuous recovered stress introduced in (20) verifies (17) for $s$ defined by (23) and therefore, using the Cauchy-Schwartz inequality,

$$
\|e\|^{2} \leq \bar{a}\left(\sigma_{e}^{\star}, \sigma_{e}^{\star}\right)+2\left|\int_{\Omega} e \cdot s d \Omega\right| \leq \bar{a}\left(\sigma_{e}^{\star}, \sigma_{e}^{\star}\right)+2\|e\|_{\mathcal{L}_{2}}\|s\|_{\mathcal{L}_{2}} .
$$

where $\|\cdot\|_{\mathcal{L}_{2}}$ stands for the standard $\mathcal{L}_{2}$ norm in $\Omega$.

Moreover, the $\mathcal{L}_{2}$ norm is bounded from above by the energy norm affected by a constant,
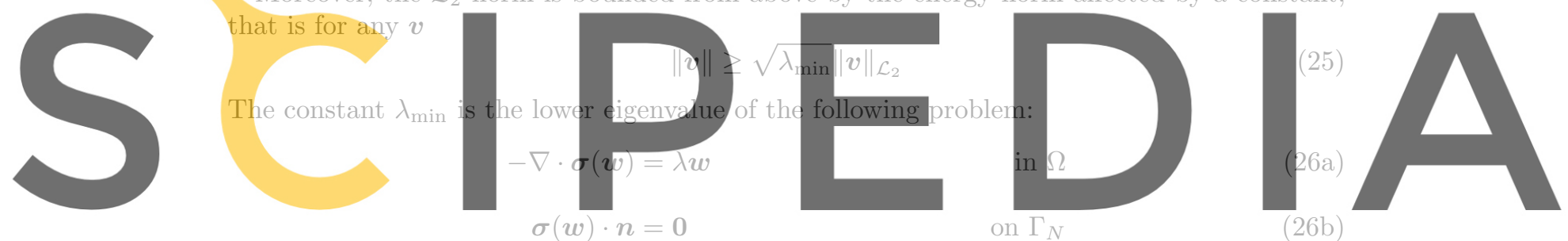

Register for free at https//www.scipedia.com to download the persion without the watermark

The weak form of the eigenvalue problem problem reads: find $\lambda \in \mathbb{R}$ and $w \in \mathcal{V}$ such that

$$
a(\boldsymbol{w}, \boldsymbol{v})=\lambda(\boldsymbol{w}, \boldsymbol{v}), \text { for all } \boldsymbol{v} \in \mathcal{V},
$$

where $(\cdot, \cdot)$ is the standard inner product in $\mathcal{L}_{2}$. The inequality $(25)$ follows from $(27)$ taking $\boldsymbol{w}=\boldsymbol{v}$ and considering that the eigenvalues are all positive.

Substituting (25) with $\boldsymbol{v}=\boldsymbol{e}$ in (24), one gets

$$
\|\boldsymbol{e}\|^{2} \leq \bar{a}\left(\boldsymbol{\sigma}_{e}^{\star}, \boldsymbol{\sigma}_{e}^{\star}\right)+\frac{2\|s\|_{\mathcal{L}_{2}}}{\sqrt{\lambda_{\min }}}\|\boldsymbol{e}\| .
$$

The scalar coefficient $\alpha=2\|s\|_{\mathcal{L}_{2}} / \sqrt{\lambda_{\min }}$ has the dimensions of the energy norm. A strict upper bound of $\|e\|$ is easily derived from (28):

$$
\|\boldsymbol{e}\| \leq E_{U B}^{\lambda_{\min }}:=\sqrt{\bar{a}\left(\boldsymbol{\sigma}_{e}^{\star}, \boldsymbol{\sigma}_{e}^{\star}\right)+\alpha^{2}}+\alpha .
$$

Note that for small $s, \alpha$ is close to zero and consequently the estimate

$$
E_{\text {est }}:=\sqrt{\bar{a}\left(\boldsymbol{\sigma}_{e}^{\star}, \boldsymbol{\sigma}_{e}^{\star}\right)}
$$

is expected to be a good approximation of $\|\boldsymbol{e}\|$. 
The value of $E_{U B}^{\lambda_{\min }}$ is easily computed from the local recovered stresses $\boldsymbol{\sigma}_{i}^{\star}, i=1, \ldots, \mathrm{n}_{\mathrm{poin}}$, except for the value of $\lambda_{\min }$. Note, however, that $\sqrt{\lambda_{\min }}$ is precisely the natural frequency of the structure and it happens to be approximated very accurately even with a coarse mesh. In fact, $\sqrt{\lambda_{\min }}$ depends on the geometry of $\Omega$, the essential boundary conditions (1c) and the material properties. The value of $\sqrt{\lambda_{\min }}$ is used as a reliable lower bound of $\|\boldsymbol{e}\| /\|\boldsymbol{e}\|_{\mathcal{L}_{2}}$, see (25). This value is nevertheless pessimistic (it is a lower bound for every $\boldsymbol{v}$ ) and leads to values of $\alpha$ that may be much larger than the actual value of $2\|s\|_{\mathcal{L}_{2}}\|\boldsymbol{e}\|_{\mathcal{L}_{2}} /\|\boldsymbol{e}\|$.

\subsection{Estimation of $\|\boldsymbol{e}\|_{\mathcal{L}_{2}}$}

The numerical experiments show that the use of (25) in the evaluation of an upper bound of $\|e\|_{\mathcal{L}_{2}}$ produces extremely large overestimations of the error in energy norm. Therefore, these values cannot be considered as accurate upper bounds.

The adaptive refinement process is driven by the estimate given in (30). Thus, the sequence of meshes is designed without using the upper bound estimates. A sequence of solutions $\boldsymbol{u}_{(i)}^{H}$, $i=1 \ldots N$, of increasing accuracy is obtained corresponding to a series of nested meshes. All this information may be used to properly assess the value of $\|e\|_{\mathcal{L}_{2}}$.

Instead of using the true upper bound of $\|e\|_{\mathcal{L}_{2}}$ that would be given by (25), a sharp estimation of this value is used in (24) to obtain an accurate upper bound of the error in
energy norm.
The displacements field $u_{(\mathrm{N})}^{H}$ obtaingd in the last mesh of the sequence con pe considered
as an approximation to the exact solution, $u_{(N)}^{H} \approx \boldsymbol{u}$. An approximation to the exact error of
the displacement fidd for the first $N$ meshes of the sequence is given by
\[ \boldsymbol{e}_{(i)}:=u-u_{(i)}^{H} \approx \boldsymbol{u}_{(N)}^{H}-\boldsymbol{u}_{(i)}^{H} \]

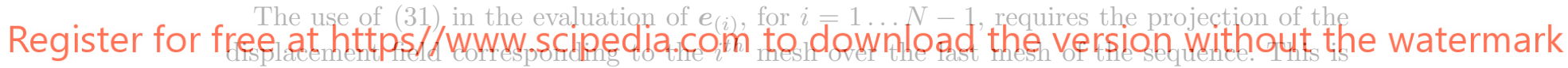

a standard data interpolation technique, especially simple and efficient if nested meshes are used.

Once the estimation of the error of the displacement field has been obtained and the corresponding $\left\|\boldsymbol{e}_{(i)}\right\|_{\mathcal{L}_{2}}$ are computed for $i=1 \ldots N-1$, the value of $\left\|\boldsymbol{e}_{(N)}\right\|_{\mathcal{L}_{2}}$ is evaluated by extrapolation from the value of $\left\|\boldsymbol{e}_{(N-1)}\right\|_{\mathcal{L}_{2}}$. This is done taking into account the number of degrees of freedom corresponding to meshes $N-1$ and $N$ and the rate of convergence of the error in the $\mathcal{L}_{2}$ norm.

Note that the rate of convergence in the $\mathcal{L}_{2}$ norm is larger than in the energy norm. In practice the estimate for $\|\boldsymbol{e}\|_{\mathcal{L}_{2}}$ is very accurate. It can be considered almost exact, although it cannot be taken as a guaranteed upper bound.

\subsection{Proposed error estimation procedures}

The error estimation procedures introduced above are summarized in figures 1 and 2. The ideas introduced in sections 4.3 and 4.4 differ in the way of evaluating the influence of the equilibrium defects and, in particular, in the assessment of $\|\boldsymbol{e}\|_{\mathcal{L}_{2}}$, see equation (24).

The first strategy, introduced in section 4.3 and illustrated in figure 1, uses the value of $\lambda_{\text {min }}$ to obtain a pessimistic bound for $\|\boldsymbol{e}\|_{\mathcal{L}_{2}}$. This strategy yields upper bounds with a large overestimation. The alternative approach described in section 4.4 is illustrated in figure 2 . The 
Step 1.- Compute $\boldsymbol{u}^{H} \in \mathcal{V}^{H}$.

Step 2.- Local recovery: compute $\boldsymbol{\sigma}_{i}^{\star}$ in every $\omega_{i}, i=1, \ldots, \mathrm{n}_{\text {poin }}$, as a local least-squares projection of $\boldsymbol{\sigma}\left(\boldsymbol{u}^{H}\right)$, enforcing equilibrium and compatibility

Step 3.- Compute continuous stress field $\boldsymbol{\sigma}^{\star}=\sum_{i=1}^{\mathrm{n}_{\mathrm{poin}}} N_{i} \boldsymbol{\sigma}_{i}^{\star}$

Step 4.- Compute equilibrium defects introduced in Step 3: $\boldsymbol{s}=\sum_{i=1}^{\mathrm{n}_{\text {poin }}}\left(\boldsymbol{\sigma}_{A}^{\star}-\boldsymbol{\sigma}_{i}^{\star}\right) \cdot \nabla N_{i}$

Step 5.- Compute the eigenvalue $\lambda_{\min }$ and $\alpha=2\|s\|_{\mathcal{L}_{2}} / \sqrt{\lambda_{\min }}$

Step 6.- Compute $E_{\text {est }}:=\sqrt{\bar{a}\left(\boldsymbol{\sigma}_{e}^{\star}, \boldsymbol{\sigma}_{e}^{\star}\right)}$ (sharp but not guaranteed upper bound) and $E_{U B}^{\lambda_{\min }}:=\sqrt{\bar{a}\left(\boldsymbol{\sigma}_{e}^{\star}, \boldsymbol{\sigma}_{e}^{\star}\right)+\alpha^{2}}+\alpha$ (guaranteed upper bound, not sharp)

Figure 1. Computing error bounds using $\lambda_{\min }$

Step 1.- Compute sequence of adaptive solutions $\boldsymbol{u}_{(j)}^{H}, j=1 \ldots N$ (using $E_{\text {est }}$ (Step 5) to adapt)

Step 2.- Local recovery: for every adaptive step (for $j=1 \ldots N$ ) compute $\boldsymbol{\sigma}_{i}^{\star}$ in every $\omega_{i}$, $i=1, \ldots, \mathrm{n}_{\text {poin }}$, as a local least-squares projection of $\boldsymbol{\sigma}\left(\boldsymbol{u}_{(j)}^{H}\right)$, enforcing equilibrium and compatibility

Step 3.- Compute continuous stress field: $\boldsymbol{\sigma}^{\star}=\sum_{i=1}^{\mathrm{n}_{\mathrm{poin}}} N_{i} \boldsymbol{\sigma}_{i}^{\star}$

Step 4.- Compute equilibrium defects introduced in Step 3: $\boldsymbol{s}=\sum_{i=1}^{\mathrm{n}_{\mathrm{poin}}}\left(\boldsymbol{\sigma}_{A}^{\star}-\boldsymbol{\sigma}_{i}^{\star}\right) \cdot \nabla N_{i}$

Step 5.- Compute $E_{\text {est }}:=\sqrt{\bar{a}\left(\boldsymbol{\sigma}_{e}^{\star}, \boldsymbol{\sigma}_{e}^{\star}\right)}$ (sharp but not guaranteed upper bound)

Step 6.- Estimate $\left\|\boldsymbol{e}_{(j)}\right\|_{\mathcal{L}_{2}}$ from the adaptive sequence

Step 7.- Compute the sharp and practical upper bound

$$
E_{U B}=\sqrt{\bar{a}\left(\boldsymbol{\sigma}_{e}^{\star}, \boldsymbol{\sigma}_{e}^{\star}\right)+2\left\|\boldsymbol{e}_{(j)}\right\|_{\mathcal{L}_{2}}\|\boldsymbol{s}\|_{\mathcal{L}_{2}}}
$$

Figure 2. Computing error bounds using the estimate for $\left\|\boldsymbol{e}_{(i)}\right\|_{\mathcal{L}_{2}}$ in a sequence of adapted solutions

idea is to build up a sequence of adapted meshes and then estimating $\|\boldsymbol{e}\|_{\mathcal{L}_{2}}$ by simply using an extrapolation technique. The adaptivity is performed using the estimate $E_{\text {est }}$, which is not a guaranteed upper bound of the error but it is a good approximation of the error. Thus, a sharp upper bound is computed.

The numerical results presented in the next section show the accuracy of the proposed procedure. 


\section{Numerical examples}

The proposed technique has been implemented in an $h$-adaptive analysis FE code based on element splitting. Non-conforming meshes are allowed (with hanging nodes) and $\mathcal{C}^{0}$ continuity is enforced using multi-point constraints (MPC's). Six-noded quadratic triangular elements are used in the numerical analyses of the following test problems: 1) $2 \times 2$ square with $3^{\text {rd }}$ order polynomial solution, 2) $L$-shaped domain under Mode I loads, 3) Thick-walled cylinder subjected to an internal pressure and 4) Gravity dam.

In the cases where an $h$-adapted sequence of meshes has been used in the numerical analysis, the $h$-adaptive process has been guided by the estimate of the error energy norm $E_{e s t}:=\sqrt{\bar{a}\left(\sigma^{\star}, \sigma^{\star}\right)}$ (the upper bound estimate is not used in the $h$-adaptive remeshing).

\subsection{Example 1: $2 \times 2$ square with with $3^{\text {rd }}$ order polynomial solution}

The $2 \times 2$ square specimen depicted in Figure 3 is analyzed. The problem setup is such that the exact solution (displacement field) is given by

$$
\begin{aligned}
& u(x, y)=x+x^{2}-2 x y+x^{3}-3 x y^{2}+x^{2} y \\
& v(x, y)=-y-2 x y+y^{2}-3 x^{2} y+y^{3}-x y^{2}
\end{aligned}
$$

The exact values of boundary traction are imposed along the edges marked with a dashed line.

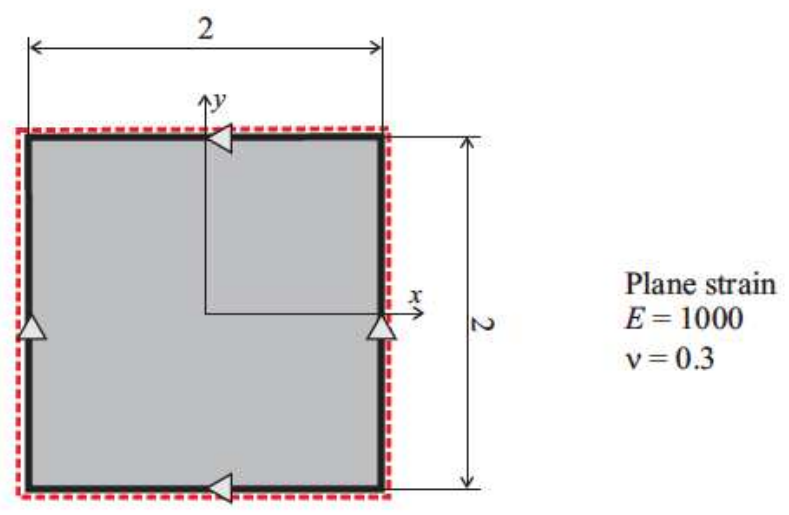

Figure 3. Example 1: $2 \times 2$ square model

The exact expressions for the components of the stress tensor are the following (in plane strain):

$$
\begin{aligned}
& \sigma_{x x}(x, y)=\frac{E}{1+\nu}\left(1+2 x-2 y+3 x^{2}-3 y^{2}+2 x y\right) \\
& \sigma_{y y}(x, y)=\frac{-E}{1+\nu}\left(1+2 x-2 y+3 x^{2}-3 y^{2}+2 x y\right) \\
& \sigma_{x y}(x, y)=\frac{E}{1+\nu}\left(-x-y+\frac{x^{2}}{2}-\frac{y^{2}}{2}-6 x y\right)
\end{aligned}
$$


Thus, the body forces ensuring equilibrium are

$$
\begin{aligned}
& b_{x}(x, y)=\frac{-E}{1+\nu}(1+y) \\
& b_{y}(x, y)=\frac{-E}{1+\nu}(1-x)
\end{aligned}
$$

Note that due to the simplicity of the expressions corresponding to body forces $\boldsymbol{b}$ and imposed tractions $\boldsymbol{t}$, the recovered stress field $\boldsymbol{\sigma}^{\star}$ obtained by using the SPR-C technique [25] is statically admissible and exactly satisfies equations (9).

A sequence of 4 uniformly-refined meshes (Figure 4) and a sequence of $4 h$-adapted meshes (Figure 5) are used in the analyses.
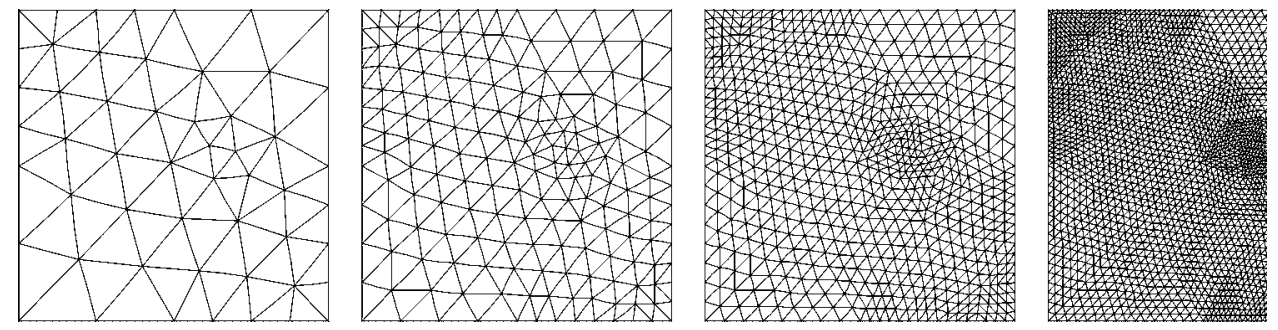

Figure 4. Example 1: Sequence of uniformly refined meshes


Figure 5. Example 1: Sequence of $h$-adapted meshes.

Tables I and II show the numerical results corresponding to the cases of uniform and $h$ adaptive refinements. The data displayed in these tables include:

- the number of degrees of freedom in each mesh, dof,

- the FE energy norm, $U=\sqrt{a\left(\boldsymbol{u}^{H}, \boldsymbol{u}^{H}\right)}$,

- the estimate of the error energy norm, $E_{\text {est }}:=\sqrt{\bar{a}\left(\boldsymbol{\sigma}^{\star}, \boldsymbol{\sigma}^{\star}\right)}$,

- the exact error energy norm, $E:=\sqrt{a(\boldsymbol{e}, \boldsymbol{e})}$,

- the effectivity index, $\theta:=E_{\text {est }} / E$,

- the estimate of the error $\mathcal{L}_{2}$ norm, $E_{\text {est }}^{\mathcal{L}_{2}}$,

- the value of $\|s\|_{\mathcal{L}_{2}}$,

- the computed upper bound for the error energy norm, $E_{U B}$, 


\begin{tabular}{ccccccccc}
\hline dof & $U$ & $E_{\text {est }}$ & $E$ & $\theta$ & $E_{\text {est }}^{\mathcal{L}_{2}}$ & $\|s\|_{\mathcal{L}_{2}}$ & $E_{U B}$ & $\theta_{U B}$ \\
\hline 366 & 253.2187 & 4.14201 & 4.12488 & 1.0042 & 0.0098498 & 71.0026 & 4.30754 & 1.0443 \\
1374 & 253.2501 & 1.06844 & 1.06338 & 1.0048 & 0.0012215 & 24.9431 & 1.09659 & 1.0312 \\
5310 & 253.2522 & 0.27201 & 0.27056 & 1.0054 & 0.0001429 & 8.9209 & 0.27666 & 1.0225 \\
20862 & 253.2523 & 0.06865 & 0.06826 & 1.0056 & 0.0000183 & 3.4437 & 0.06956 & 1.0190 \\
\hline
\end{tabular}

Table I. Example 1: Uniform refinement. Numerical results

\begin{tabular}{ccccccccc}
\hline dof & $U$ & $E_{\text {est }}$ & $E$ & $\theta$ & $E_{\text {est }}^{\mathcal{L}_{2}}$ & $\|s\|_{\mathcal{L}_{2}}$ & $E_{U B}$ & $\theta_{U B}$ \\
\hline 366 & 253.21875 & 4.142010 & 4.124888 & 1.0042 & 0.0098496 & 71.0026 & 4.307545 & 1.0443 \\
1228 & 253.24963 & 1.176203 & 1.172519 & 1.0031 & 0.0014005 & 38.7167 & 1.221433 & 1.0417 \\
4658 & 253.25217 & 0.305669 & 0.304719 & 1.0031 & 0.0001729 & 19.7589 & 0.316650 & 1.0392 \\
18092 & 253.25234 & 0.078248 & 0.077971 & 1.0035 & 0.0000226 & 9.0089 & 0.080807 & 1.0364 \\
\hline
\end{tabular}

Table II. Example 1: $h$-adaptive refinement. Numerical results

- the upper bound effectivity index, $\theta_{U B}:=E_{U B} / E$

The evolution of the effectivity indices $\theta$ and $\theta_{U B}$ is graphically represented in Figures 6 and 7. The results of the analyses show the high accuracy of both the proposed error estimator

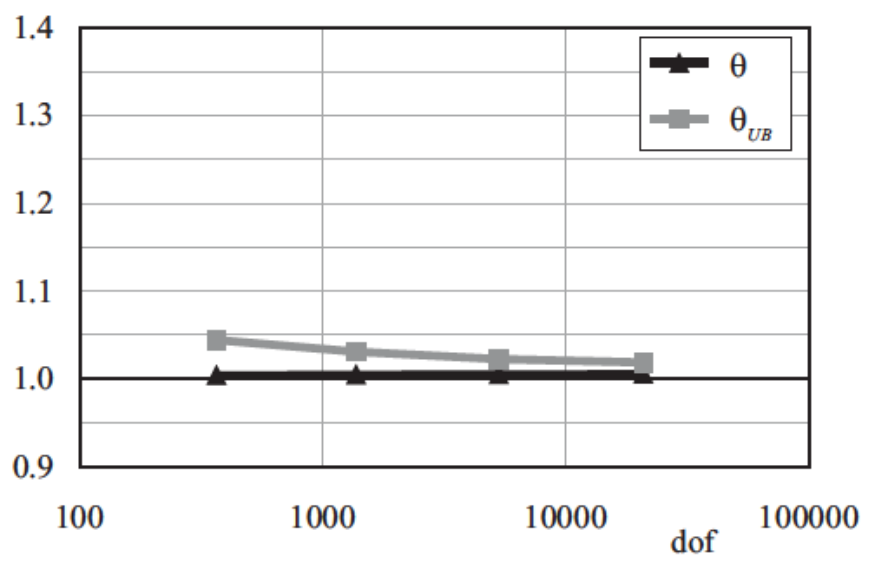

Figure 6. Example 1: Uniform refinement. Effectivity indices $\theta$ and $\theta_{U B}$

in energy norm $E_{e s t}$ and the corresponding error bound $E_{U B}$. Moreover, the accuracy of the estimates is preserved at the local level. The local effectivity is represented in Figure 8 by the quantity $D$ defined element by element as

$$
\begin{array}{lr}
D=\theta^{e}-1 & \text { if } \theta^{e} \geq 1 \\
D=1-1 / \theta^{e} & \text { if } \theta^{e}<1,
\end{array}
$$

where $\theta^{e}$ is the local counterpart (associated with element $e$ ) of $\theta$. Index $D$ is particulary well suited for the local representation of the effectivity index because it fairly compares the 




Figure 7. Example 1: $h$-adaptive refinement. Effectivity indices $\theta$ and $\theta_{U B}$

underestimation of the error $(D<0)$ and the overestimation $(D>0)$. The good behavior of the estimates results on values of $D$ close to zero.

Figure 8 displays the distribution of $D$ in the sequence of uniformly refined meshes. Note that the values of $D$ are in all the cases very small, that is the estimate is performing very well also at the local level.
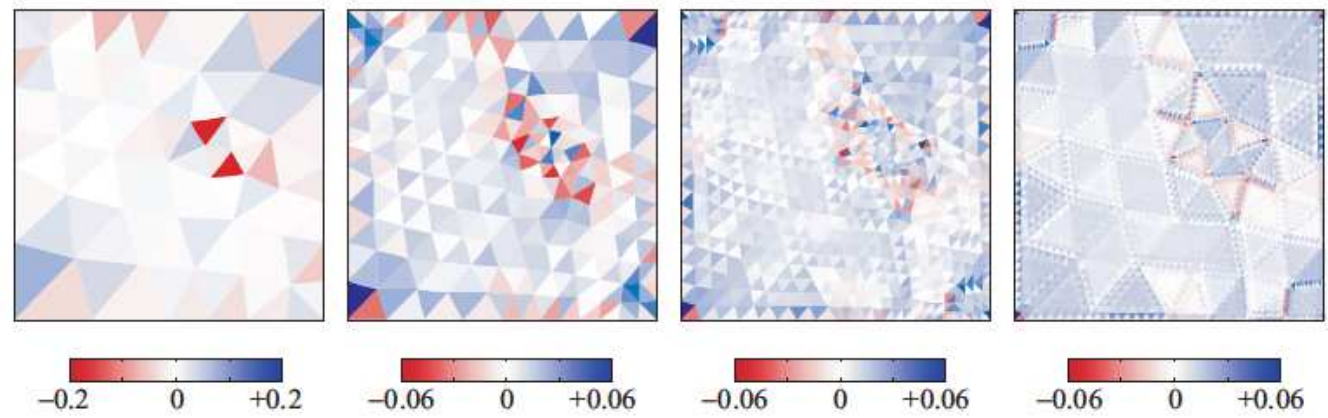

Figure 8. Example 1: uniform refinement. Distribution of index $D$.

Figure 9 shows the distribution of the local contributions to $\|s\|_{\mathcal{L}_{2}}$. Recall that $E_{\text {est }}$ cannot be proved to be an upper bound of the error unless $\|s\|_{\mathcal{L}_{2}}$ is negligible. It is worth noting that the values of $\|s\|_{\mathcal{L}_{2}}$ decrease along the remeshing process but they are far from being negligible. Moreover, the local distribution is not uniform and therefore pollutes the local behavior of the estimate in different zones of the domain.

The results previously presented are based on the estimation of the error in the $\mathcal{L}_{2}$ norm exposed in section 4.4. Table III shows the results that would be obtained if (25) was used in the evaluation of an upper bound of $\|e\|_{\mathcal{L}_{2}}$. The data displayed in the table include:

- the number of degrees of freedom in each mesh, dof, 

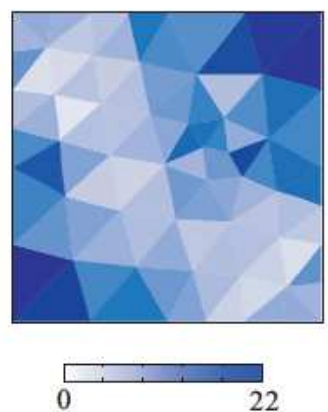
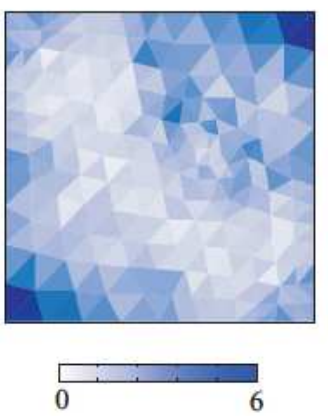

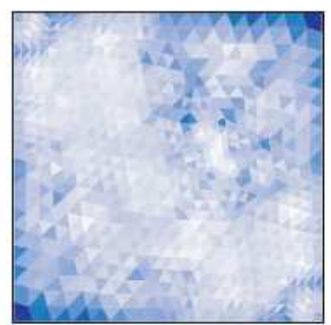

0


Figure 9. Example 1: uniform refinement. Distribution of $\|s\|_{\mathcal{L}_{2}}$.

- the estimated error in the $\mathcal{L}_{2}$ norm, $E_{\text {est }}^{\mathcal{L}_{2}}$ (see section 4.4),

- the exact error in the $\mathcal{L}_{2}$ norm, $E^{\mathcal{L}_{2}}$,

- the effectivity index $\theta_{\mathcal{L}_{2}}:=E_{\text {est }}^{\mathcal{L}_{2}} / E^{\mathcal{L}_{2}}$,

- the lower eigenvalue of the problem given in (26), $\lambda_{\min }$,

- the exact value of $\|e\|^{2} /\|e\|_{\mathcal{L}_{2}}^{2}, \lambda_{\text {exa }}$,

- the upper bound of the error in the $\mathcal{L}_{2}$ norm obtained using $\lambda_{\min }, E_{\lambda_{\min }}^{\mathcal{L}_{2}}$,

- the effectivity index $\theta_{\mathcal{L}_{2}}^{\lambda_{\min }}:=E_{\lambda_{\min }}^{\mathcal{L}_{2}} / E^{\mathcal{L}_{2}}$,

- the upper bound of the error in energy norm obtained using $E_{\lambda_{\min }}^{\mathcal{L}_{2}}, E_{U B}^{\lambda_{\min }}$

- the upper bound effectivity index, $\theta_{U B}^{\lambda_{\min }}:=E_{U B}^{\lambda_{\min }} / E$

\begin{tabular}{cccccccccc}
\hline dof & $E_{\text {est }}^{\mathcal{L}_{2}}$ & $E^{\mathcal{L}_{2}}$ & $\theta_{\mathcal{L}_{2}}$ & $\lambda_{\min }$ & $\lambda_{\text {exa }}$ & $E_{\lambda_{\min }}^{\mathcal{L}_{2}}$ & $\theta_{\mathcal{L}_{2}}^{\lambda_{\min }}$ & $E_{U B}^{\lambda_{\min }}$ & $\theta_{U B}^{\lambda_{\min }}$ \\
\hline 366 & 0.009850 & 0.009138 & 1.078 & 262.23 & $2.0 \times 10^{5}$ & 299.054 & 32726.3 & 17.7715 & 4.308 \\
1374 & 0.001222 & 0.001137 & 1.074 & 217.79 & $8.7 \times 10^{5}$ & 102.205 & 89897.7 & 6.9152 & 6.503 \\
5310 & 0.000143 & 0.000139 & 1.028 & 185.99 & $3.8 \times 10^{6}$ & 36.065 & 259275.1 & 2.7166 & 10.041 \\
20862 & 0.000018 & 0.000017 & 1.070 & 162.17 & $1.6 \times 10^{7}$ & 13.830 & 806210.3 & 1.1418 & 16.725 \\
\hline
\end{tabular}

Table III. Example 1: uniform refinement. Numerical results with upper bound of $\|e\|_{\mathcal{L}_{2}}$ computed from $\lambda_{\min }$.

The comparison of the upper bound effectivity indices $\theta_{U B}$ (see Table I) and $\theta_{U B}^{\lambda_{\min }}$ (see Table III) clearly shows that the performance of $E_{U B}$ is superior to the performance of $E_{U B}^{\lambda_{\min }}$. The reason behind this difference is that the values of $E_{\text {est }}^{\mathcal{L}_{2}}$ used in the evaluation of $E_{U B}$ are quite accurate whereas the values of $E_{\lambda_{\min }}^{\mathcal{L}_{2}}$ used in the evaluation of $E_{U B}^{\lambda_{\min }}$ would even increase their inaccuracy as the mesh is refined (see values of $\theta_{\mathcal{L}_{2}}$ and $\theta_{\mathcal{L}_{2}}^{\lambda_{\min }}$ in Table III).

In the two examples previously presented, the values of $E_{\text {est }}$ are very sharp overestimations of $E$, however, under some circumstances the value of $E_{\text {est }}$ can underestimate the true error. Table IV shows the results obtained with another $h$-adaptive refinement. The only difference between this new refinement and the previous one is the amount of error reduction requested for the generation of the new meshes. Note that in the $3^{r d}$ mesh (values represented in italics) $E_{e s t}$ slightly underestimates the value of $E$. Notwithstanding, the value of $E_{U B}$ is always above E. 


\begin{tabular}{ccccccccc}
\hline dof & $U$ & $E_{\text {est }}$ & $E$ & $\theta$ & $E_{\text {est }}^{\mathcal{L}_{2}}$ & $\|s\|_{\mathcal{L}_{2}}$ & $E_{U B}$ & $\theta_{U B}$ \\
\hline 366 & 253.218754 & 4.142010 & 4.124888 & 1.0042 & 0.00984940 & 71.0026 & 4.307541 & 1.0443 \\
1088 & 253.248492 & 1.401329 & 1.397618 & 1.0027 & 0.00186969 & 58.7963 & 1.477696 & 1.0573 \\
3298 & 253.251822 & 0.516379 & 0.516523 & 0.9997 & 0.00038181 & 42.4774 & 0.546886 & 1.0588 \\
8418 & 253.252266 & 0.205664 & 0.205288 & 1.0018 & 0.00007531 & 22.9018 & 0.213886 & 1.0419 \\
20126 & 253.252338 & 0.075170 & 0.074824 & 1.0046 & 0.00002037 & 10.8454 & 0.078054 & 1.0432 \\
\hline
\end{tabular}

Table IV. Example 1: $h$-adaptive refinement. Numerical results

\subsection{Example 2: L-shaped domain under Mode I loads}

A $L$-shaped portion of an infinite domain has been loaded with the stress components of the first symmetric (Mode I) term of the asymptotic expansion of the exact solution around the vertex, see Figure 10. As in the previous example, the exact values of boundary traction over the dashed edges are prescribed.

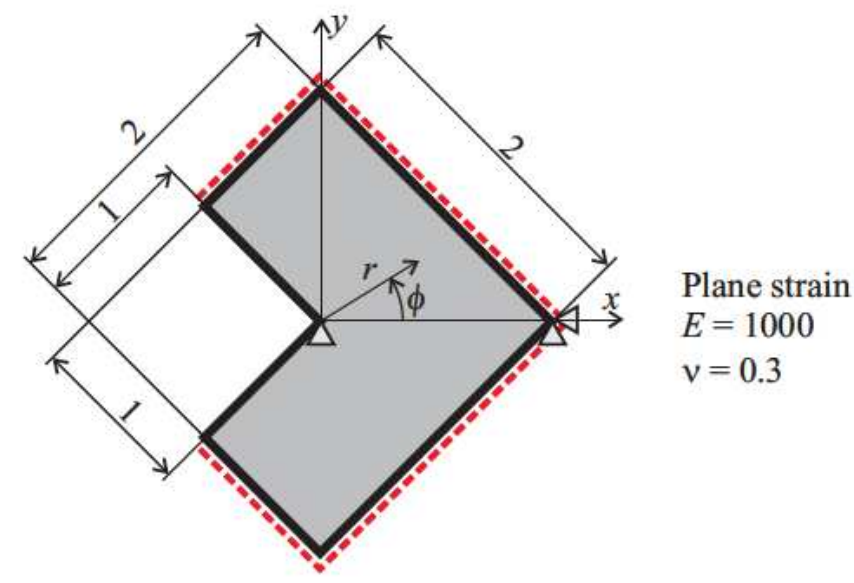

Figure 10. Example 2: $L$-shaped domain in Mode I. Model

Details about the exact solution in the neighborhoods of singular points, and, in particular, the exact solution for this singular stress field problem, with body load $\boldsymbol{b}=\mathbf{0}$, can be obtained from [30]. This exact solution is given by

$$
\begin{aligned}
\sigma_{x x} & =\lambda r^{\lambda-1}[(2-Q(\lambda+1)) \cos ((\lambda-1) \phi)-(\lambda-1) \cos ((\lambda-3) \phi)] \\
\sigma_{y y} & =\lambda r^{\lambda-1}[(2+Q(\lambda+1)) \cos ((\lambda-1) \phi)+(\lambda-1) \cos ((\lambda-3) \phi)] \\
\sigma_{x y} & =\lambda r^{\lambda-1}[(Q(\lambda+1)) \sin ((\lambda-1) \phi)+(\lambda-1) \sin ((\lambda-3) \phi)]
\end{aligned}
$$

where $r$ and $\phi$ are the polar coordinates and, up to the accuracy required in the computations, the parameters $\lambda$ and $Q$ for this load conditions take the values $\lambda=0.544483736782464$ and $Q=0.543075578836737$.

In this case it is easy to impose equilibrium to the recovered stresses $\sigma^{\star}$, that is equation (9a), because $\boldsymbol{b}=\mathbf{0}$. Nevertheless, the boundary equilibrium, see equation (9b), is exactly satisfied 
only at nodal points. Along the rest of the boundary this equation is only approximately satisfied because the stress recovery functions considered in each patch are $2^{\text {nd }}$ order polynomials and the boundary traction is a trigonometric function. Thus, the SPR-C technique giving $\boldsymbol{\sigma}^{\star}$ prescribes (9b) approximating $\boldsymbol{t}$ by a $2^{\text {nd }}$ order Taylor's expansion around the patch assembly nodes. The results obtained using this approximation are found to be very accurate.

Figure 11 shows the sequence of $h$-adapted meshes obtained during the analysis of this problem. Table $\mathrm{V}$ displays the numerical results of the analysis showing the high accuracy of $E_{\text {est }}$ even for this singular problem. Note, however, that $E$ is underestimated by $E_{\text {est }}$ in the last mesh of the sequence. This is because, in this case, the presence of the singularity produces an increase of $\|s\|_{\mathcal{L}_{2}}$ with the refinement of the model, resulting in an inaccurate value of $E_{U B}$.

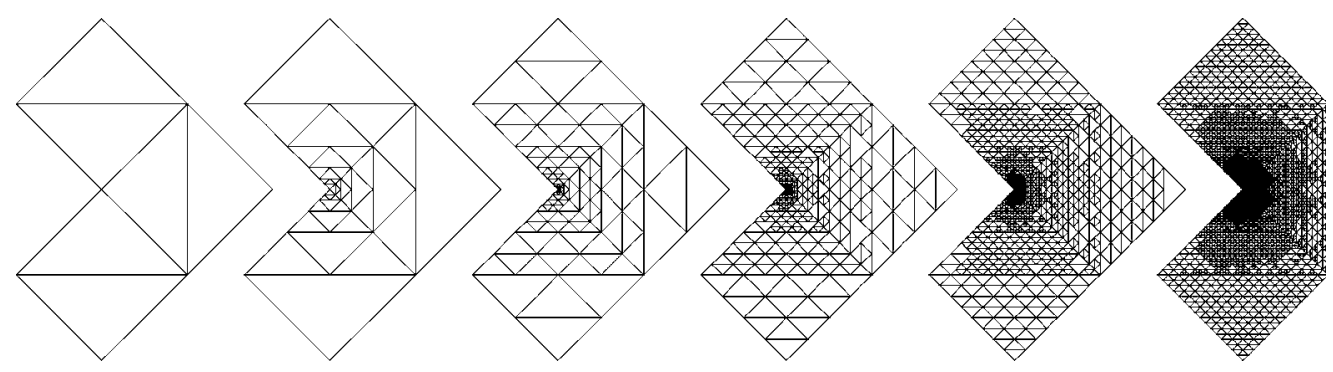

Figure 11. Example 2: Sequence of $h$-adapted meshes

\begin{tabular}{ccccccccc}
\hline dof & $U$ & $E_{\text {est }}$ & $E$ & $\theta$ & $E_{\text {est }}^{\mathcal{L}_{2}}$ & $\|\boldsymbol{s}\|_{\mathcal{L}_{2}}$ & $E_{U B}$ & $\theta_{U B}$ \\
\hline \hline 39 & 0.0865196 & 0.040676 & 0.026063 & 1.5607 & 0.00133454 & 2.836 & 0.09604 & 3.6851 \\
223 & 0.0904952 & 0.013913 & 0.012323 & 1.1290 & 0.00042122 & 10.474 & 0.09496 & 7.7060 \\
741 & 0.0910440 & 0.004951 & 0.004830 & 1.0251 & 0.00010816 & 37.575 & 0.09029 & 18.6940 \\
2605 & 0.0911413 & 0.001599 & 0.001591 & 1.0047 & 0.00002256 & 133.823 & 0.07772 & 48.8373 \\
8983 & 0.0911531 & 0.000463 & 0.000463 & 1.0013 & 0.00000382 & 473.861 & 0.06021 & 130.1740 \\
31461 & 0.0911541 & 0.000130 & 0.000131 & 0.9984 & 0.00000058 & 1680.164 & 0.04428 & 339.2138 \\
\hline
\end{tabular}

Table V. Example 2: $h$-adaptive refinement. Numerical results

\subsection{Example 3: Thick-walled cylinder subjected to an internal pressure}

The geometry and loads of the problem are displayed in Figure 12. The exact solution is available and its expression in polar coordinates is the following:

$$
u_{r}=\frac{p(1+\nu)}{E\left(k^{2}-1\right)}\left[(1-2 \nu) r+\frac{b^{2}}{r}\right] \quad \text { (radial displacement) }
$$




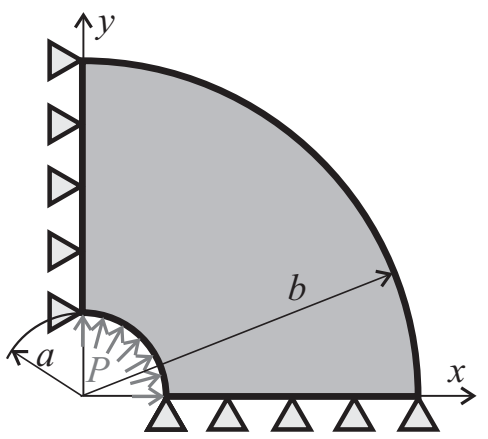

Plane strain

$a=5$

$b=20$

$P=1$

$E=1000$

$v=0.3$

Figure 12. Example 3: Cylinder subjected to internal pressure. Model.

The exact stresses are given both in polar (cylindrical) and Cartesian coordinates:

$$
\begin{aligned}
\sigma_{r}=\frac{p}{k^{2}-1}\left(1-\frac{b^{2}}{r^{2}}\right) & \sigma_{x x}=\sigma_{r} \cos ^{2}(\phi)+\sigma_{t} \sin ^{2}(\phi) \\
\sigma_{t}=\frac{p}{k^{2}-1}\left(1+\frac{b^{2}}{r^{2}}\right) & \sigma_{y y}=\sigma_{r} \sin ^{2}(\phi)+\sigma_{t} \cos ^{2}(\phi) \\
\sigma_{x y} & =\left(\sigma_{r}-\sigma_{t}\right) \sin (\phi) \cos (\phi)
\end{aligned}
$$

As in the previous example, the SPR-C technique [25] builds-up $\boldsymbol{\sigma}^{\star}$ such that equation (9a) is exactly satisfied. However, equation (9b) is only approximately satisfied. Here, the presence of curved boundaries is an additional source of error because in this kind of contours the SPR-C technique can only impose the boundary equilibrium at the nodal points.

A sequence of 4 uniformly-refined meshes (Figure 13) and a sequence of $5 h$-adapted meshes (Figure 14) have been analyzed. The results of these analyses are shown in Tables VI and VII. The evolution of the effectivity indices $\theta$ and $\theta_{U B}$ in these tables is also represented in Figures 15 and 16. Figure 17 shows the local distribution of $\|s\|_{\mathcal{L}_{2}}$. The results show a behavior similar to Example 1.
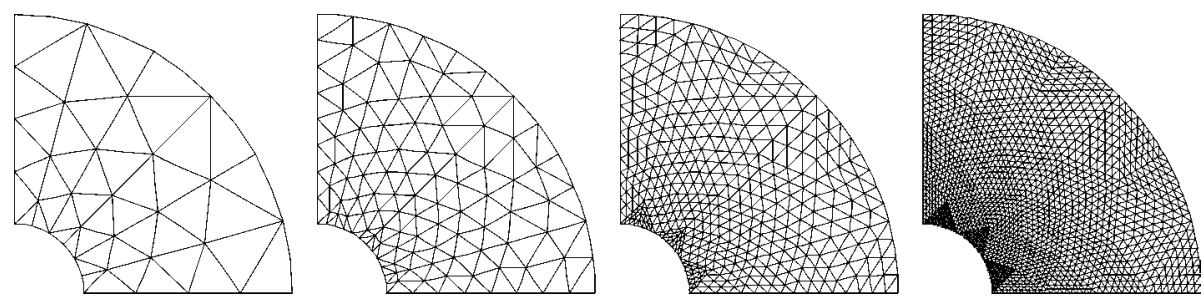

Figure 13. Example 3: Sequence of uniformly refined meshes 


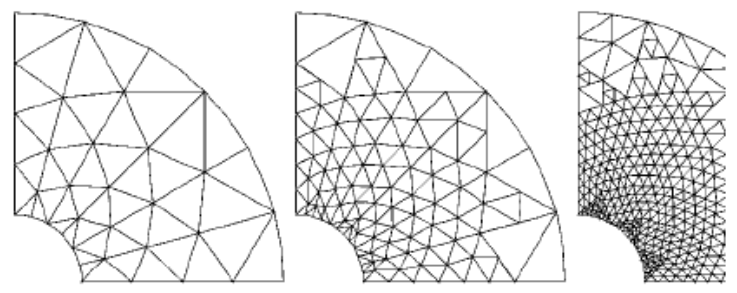

Figure 14. Example 3: Sequence of $h$-adapted meshes

\begin{tabular}{ccccccccc}
\hline dof & $U$ & $E_{\text {est }}$ & $E$ & $\theta$ & $E_{\text {est }}^{\mathcal{L}_{2}}$ & $\|\boldsymbol{s}\| \mathcal{L}_{2}$ & $E_{U B}$ & $\theta_{U B}$ \\
\hline 216 & 236.048312 & 10.665979 & 9.721769 & 1.0971 & 0.140752279 & 190.8099 & 12.941289 & 1.3312 \\
816 & 236.236755 & 2.847802 & 2.784362 & 1.0228 & 0.019200372 & 72.0128 & 3.297775 & 1.1844 \\
3168 & 236.252127 & 0.755436 & 0.747546 & 1.0106 & 0.002347526 & 23.1918 & 0.824361 & 1.1028 \\
12480 & 236.253237 & 0.194653 & 0.192868 & 1.0093 & 0.000300238 & 7.0348 & 0.205218 & 1.0640 \\
\hline
\end{tabular}

Table VI. Example 3: Uniform refinement. Numerical results

\begin{tabular}{ccccccccc}
\hline dof & $U$ & $E_{\text {est }}$ & $E$ & $\theta$ & $E_{\text {est }}^{\mathcal{L}_{2}}$ & $\|\boldsymbol{s}\|_{\mathcal{L}_{2}}$ & $E_{U B}$ & $\theta_{U B}$ \\
\hline 216 & 236.048312 & 10.665979 & 9.721769 & 1.0971 & 0.14067866 & 190.8099 & 12.940204 & 1.3311 \\
724 & 236.234717 & 3.043396 & 2.958358 & 1.0287 & 0.02989259 & 72.8848 & 3.690487 & 1.2475 \\
2732 & 236.251858 & 0.838411 & 0.830050 & 1.0101 & 0.00469713 & 23.7124 & 0.962130 & 1.1591 \\
9940 & 236.253205 & 0.230239 & 0.228504 & 1.0076 & 0.00073652 & 7.4577 & 0.252973 & 1.1071 \\
37226 & 236.252847 & 0.061748 & 0.061404 & 1.0056 & 0.00010162 & 2.5038 & 0.065740 & 1.0706 \\
\hline
\end{tabular}

Table VII. Example 3: $h$-adaptive refinement. Numerical results

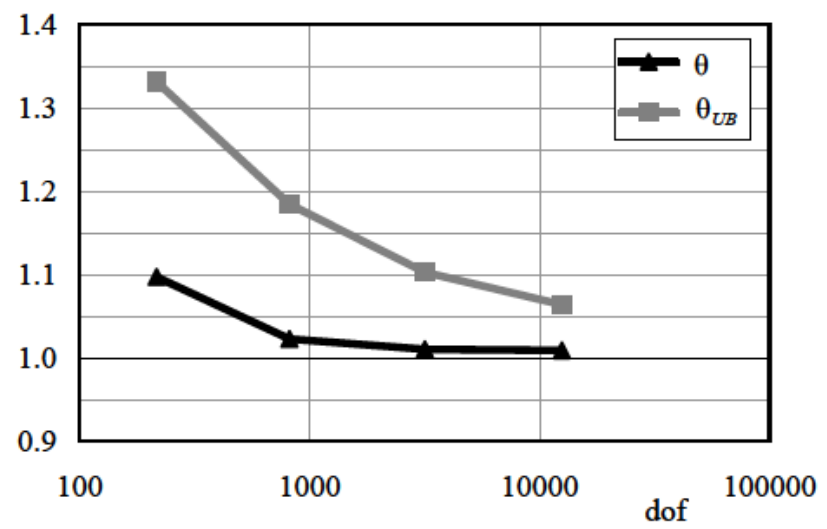

Figure 15. Example 3: Uniform refinement. Effectivity indices $\theta$ and $\theta_{U B}$

\subsection{Example 4: Gravity dam}

The gravity dam represented in Figure 18 is analyzed. The loading includes both self-weight and water pressure. The geometry has been rounded up and it does not include any sharp 


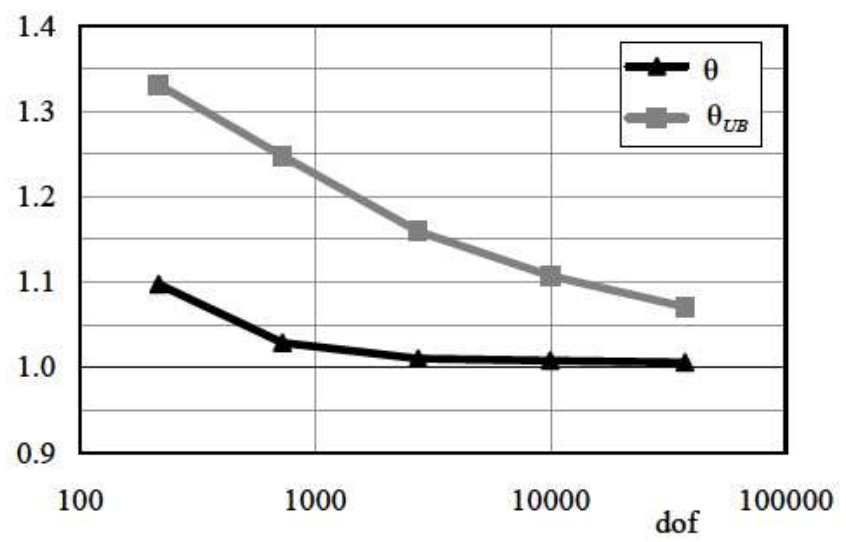

Figure 16. Example 3: $h$-adaptive refinement. Effectivity indices $\theta$ and $\theta_{U B}$
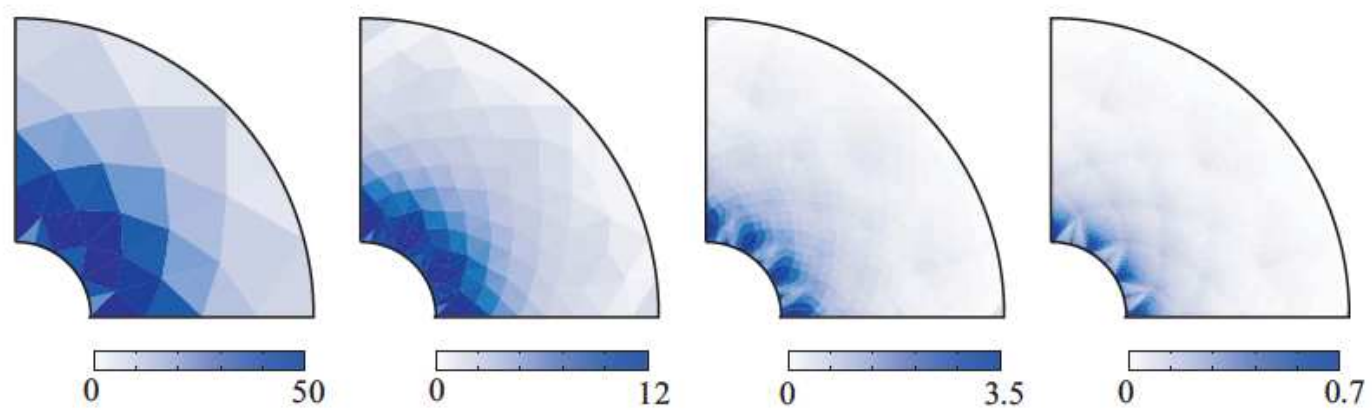

Figure 17. Example 3: $h$-adaptive refinement. Local distribution of $\|s\|_{\mathcal{L}_{2}}$

reentrant corner.

The body load $b$ is constant and therefore the recovered stress field $\sigma^{\star}$ exactly satisfies equation (9a). Nevertheless, the boundary traction, equation (9b), is only approximately satisfied due to the presence of curved boundaries. In any case, equation (9b) is exactly satisfied at the nodal points.

The sequence of $4 \mathrm{~h}$-adapted meshes displayed in Figure 19 is analyzed. Table VIII shows the numerical results obtained from the analysis. The new parameters $\eta \%$ and $\eta_{U B} \%$ included in this table respectively represent the estimated relative error in energy norm and the upper bound of the relative error. These two parameters have been represented in Figure 20.

In this example no exact solution is available and, consequently, the effectivity indices cannot be evaluated. However, the results obtained for this problem are likely similar to those obtained in the previous examples. For example, the estimated relative errors converge properly, see Figure 20, both for $E_{\text {est }}$ and the upper bound $E_{U B}$. Note that the only safe upper bound is the $E_{U B}$, even if $E_{e s t}$ is likely a sharper estimate. It is worth mentioning that in the refined meshes the over estimation introduced by $E_{U B}$ is less and less significative, compared to $E_{\text {est }}$. 


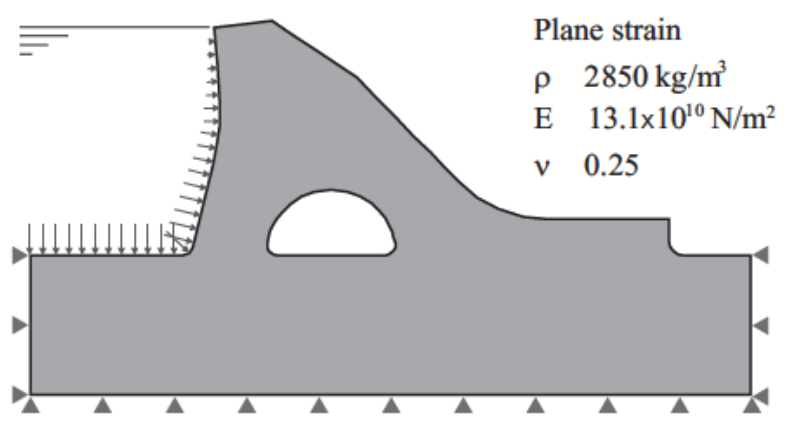

Figure 18. Example 4: Gravity dam model
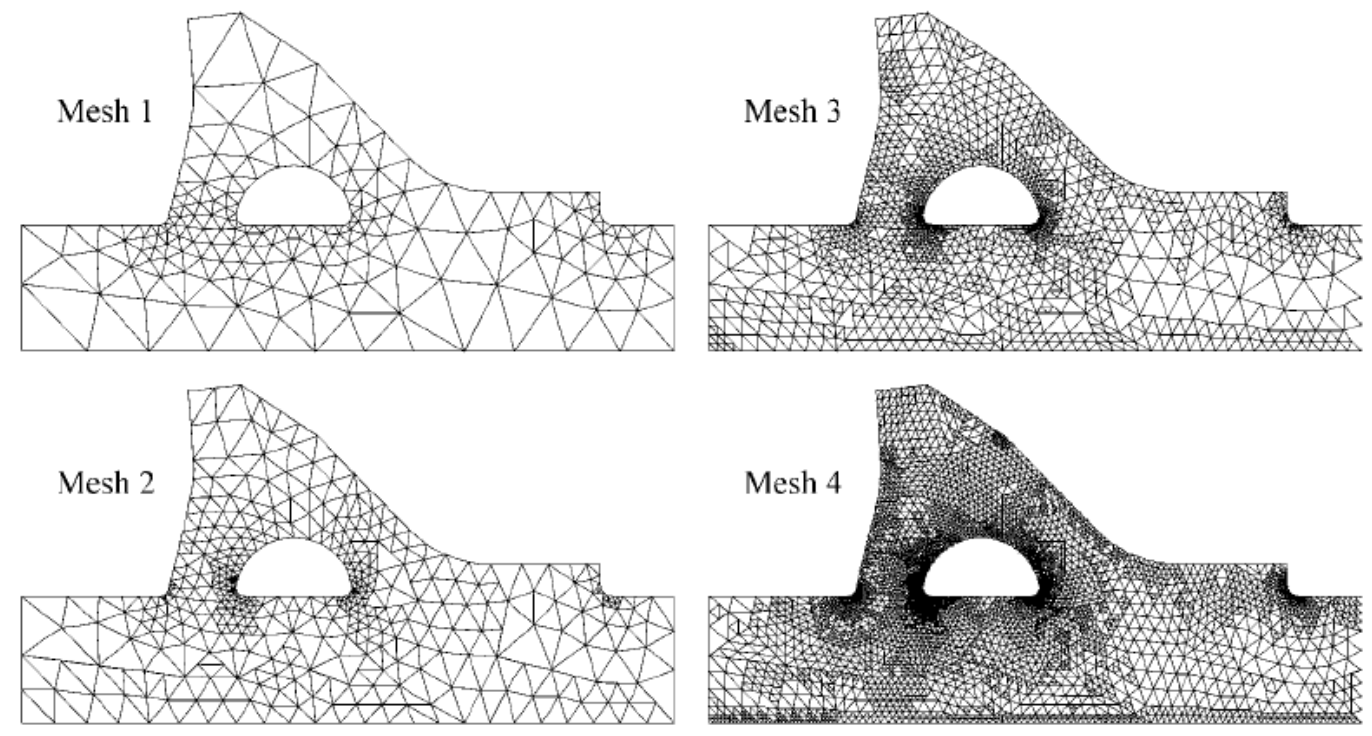

Figure 19. Example 4: Sequence of $h$-adapted meshes

\begin{tabular}{cccccccc}
\hline dof & $U$ & $E_{\text {est }}$ & $E_{\text {est }}^{\mathcal{L}_{2}}$ & $\|s\|_{\mathcal{L}_{2}}$ & $E_{U B}$ & $\eta \%$ & $\eta_{U B} \%$ \\
\hline 1327 & 93.0138676 & 4.345432 & $1.95 \times 10^{-5}$ & $1.17 \times 10^{6}$ & 8.034644 & 4.6667 & 8.6060 \\
3315 & 93.0625794 & 1.394880 & $3.16 \times 10^{-6}$ & $5.43 \times 10^{5}$ & 2.319066 & 1.4987 & 2.4911 \\
11161 & 93.0699011 & 0.434861 & $4.18 \times 10^{-7}$ & $2.58 \times 10^{5}$ & 0.636241 & 0.4672 & 0.6836 \\
37375 & 93.0706132 & 0.127222 & $6.82 \times 10^{-8}$ & $1.04 \times 10^{5}$ & 0.174210 & 0.1367 & 0.1871 \\
\hline
\end{tabular}

Table VIII. Example 4: $h$-adaptive refinement. Numerical results

\section{Conclusions}

A new strategy to compute upper bounds of the error from equilibrated recovered stresses has been introduced. With respect to previous works, this approach uses a different local 


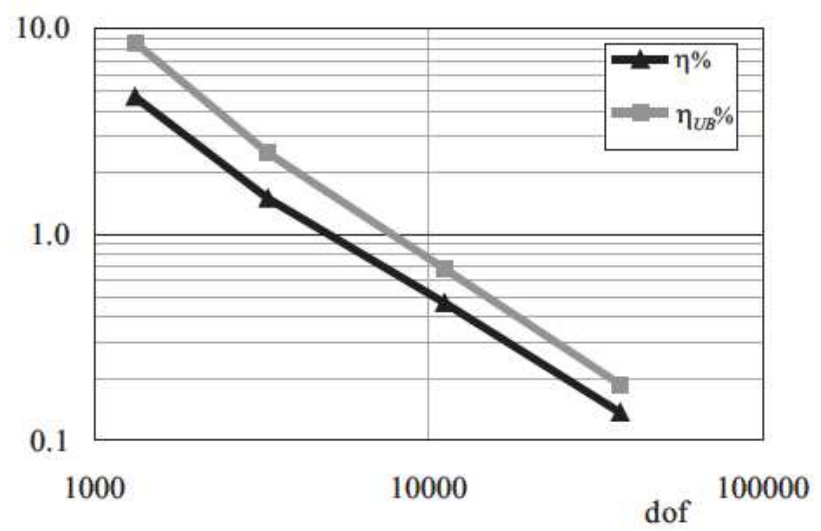

Figure 20. Example 4: Relative errors

recovery technique enforcing full statical admissibility (matching body loads and boundary tractions) and compatibility (via the kinematical admissibility). Moreover, in this work the loss of equilibrium produced when enforcing the global stress continuity is not assumed to be negligible. This assumption is removed by taking into account the equilibrium defects in the resulting estimate.

This opens a promising line of work based on exploiting the deficiencies of global equilibrium in the recovered solutions. For instance, instead of enforcing continuity and computing the equilibrium defects, an alternative approach is to use the discontinuous average equilibrated stress, $\sigma_{A}^{\star}$, and to include the stress jumps to compute an error bound using proposition 4 .

\section{ACKNOWLEDGEMENTS}

Sponsored by Ministerio de Educación y Ciencia (grants DPI2004-03000, CGL2004-06171C03-01/CLI and DPI2004-07782-C02-02), Universidad Politécnica de Valencia and Generalitat Valenciana.

\section{REFERENCES}

1. M. Ainsworth and J.T. Oden. A posteriori error estimation in finite element analysis. John Wiley \& Sons, Chichester, 2000.

2. W. Bangerth and R. Rannacher. Adaptive Finite Element Methods for Differential Equations. Birkhäuser Verlag AG, 2003.

3. O.C. Zienkiewicz and J.Z. Zhu. A simple error estimator and adaptive procedure for practical engineering analysis. International Journal for Numerical Methods in Engineering, 24:337-357, 1987.

4. O.C. Zienkiewicz and J.Z. Zhu. The superconvergent patch recovery (SPR) and adaptive finite element refinement. Computer Methods in Applied Mechanics and Engineering, 101:207-224, 1992.

5. O.C. Zienkiewicz and J.Z. Zhu. The superconvergent patch recovery (SPR) and a posteriori error estimates. Part 1: The recovery technique. International Journal for Numerical Methods in Engineering, 33:1331-1364, 1992. 
6. O.C. Zienkiewicz and J.Z. Zhu. The superconvergent patch recovery (SPR) and a posteriori error estimates. Part 2: Error estimates and adaptivity. International Journal for Numerical Methods in Engineering, 33:1365-1382, 1992.

7. I. Babuška, T. Strouboulis, and C.S. Upadhyay. A model study of the quality of a posteriori error estimators for linear elliptic problems. Error estimation in the interior of patchwise uniform grids of triangles. Computer Methods in Applied Mechanics and Engineering, 114:307-378, 1994.

8. I. Babuška, T. Strouboulis, C.S. Upadhyay, S.K. Gangaraj, and K. Copps. Validation of a posteriori error estimators by numerical approach. International Journal for Numerical Methods in Engineering, 37:1073-1123, 1994.

9. I. Babuška, T. Strouboulis, C.S. Upadhyay, S.K. Gangaraj, and K. Copps. An objective criterion for assessing the reliability of a posteriori error estimators in finite element computations. U.S.A.C.M. Bulletin, No. 7:4-16, 1994.

10. P. Díez, J.J. Egozcue, and A. Huerta. A posteriori error estimation for standard finite element analysis. Computer Methods in Applied Mechanics and Engineering, 163:141-157, 1998.

11. P. Díez, N. Parés, and A. Huerta. Recovering lower bounds of the error postprocessing implicit residual a posteriori error estimates. International Journal for Numerical Methods in Engineering, 56:1465-1488, 2003.

12. N.E. Wiberg and F. Abdulwahab. Patch recovery based on superconvergent derivatives and equilibrium. International Journal for Numerical Methods in Engineering, 36:2703-2724, 1993.

13. N.E. Wiberg, F. Abdulwahab, and S. Ziukas. Enhanced superconvergent patch recovery incorporating equilibrium and boundary conditions. International Journal for Numerical Methods in Engineering, 37:3417-3440, 1994.

14. T. Blacker and T. Belytschko. Superconvergent patch recovery with equilibrium and conjoint interpolant enhancements. International Journal for Numerical Methods in Engineering, 37:517-536, 1994.

15. A.C.A. Ramsay and E.A.W. Maunder. Effective error estimation from continuous, boundary admissible estimated stress fields. Computers and Structures, 61:331-343, 1996.

16. T. Lee T, H.C. Park, and S.W. Lee. A superconvergent stress recovery technique with equilibrium constraint. International Journal for Numerical Methods in Engineering, 40:1139-1160, 1997.

17. J. Aalto. Built-in field equations for recovery procedures. Computers and Structures, 64:157-176, 1997.

18. J. Aalto. An element by element recovery method with built-in field equations. Computers and Structures, 64:177-196, 1997.

19. J. Aalto and M. Perälä. Built-in field equations for patch recovery procedures using weighted residuals. Computers and Structures, 73:91-118, 1999.

20. J. Aalto and M. Åman. Polinomial representation for patch recovery procedures. Computers and Structures, 73:119-146, 1999.

21. B. Boroomand and O.C. Zienkiewicz. Recovery by equilibrium in patches (REP). International Journal for Numerical Methods in Engineering, 40:137-164, 1997.

22. T. Kvamsdal and K.M. Okstad. Error estimation based on superconvergent patch recovery using statically admissible stress fields. International Journal for Numerical Methods in Engineering, 42:443-472, 1998.

23. H.C. Park, S.H. Shin, and S.W. Lee. A superconvergent stress recovery technique for accurate boundary stress extraction. International Journal for Numerical Methods in Engineering, 45:1227-1242, 1999.

24. J.J. Ródenas, F.J. Fuenmayor, A. Roda, and A. Pedrosa. Modification of the SPR technique to ensure the fulfillment of imposed tractions in boundary nodes. In N.E. Wiberg and P. Díez, editors, Adaptive Modeling and Simulation, volume 1. CIMNE, October 2003.

25. J.J. Ródenas, M. Tur, F.J. Fuenmayor, and A. Vercher. Improvement of the superconvergent patch recovery technique by the use of constraint equations: the SPR-C technique. Submitted to International Journal for Numerical Methods in Engineering.

26. J.F. Abel and M.S. Shephard. An algorithm for multipoint constraints in finite element analysis. International Journal for Numerical Methods in Engineering, 14:464-467, 1979.

27. C. Farhat, C. Lacour, and D. Rixen. Incorporation of linear multipoint constraints in substructure based iterative solvers. part 1: a numerically stable algorithm. International Journal for Numerical Methods in Engineering, 43:997-1016, 1998.

28. M. Ainsworth and J.T. Oden. A procedure for a posteriori error estimation for $h-p$ finite element methods. Computer Methods in Applied Mechanics and Engineering, 101:73-96, 1992.

29. S.P. Timoshenko and J.N. Goodier. Theory of Elasticity. McGraw-Hill, New York, 2 edition, 1951.

30. B.A. Szabó and I. Babuška. Finite Element Analysis. John Wiley \& Sons, Inc, New York, 1 edition, 1991. 